\title{
Retratamiento ortodóntico con técnica de arco segmentado en mordida cruzada posterior unilateral
}

\author{
Orthodontic retreatment with segmented arch technique \\ in unilateral posterior crossbite
}

\begin{abstract}
Jennisse Katherine Soncco Quispe ${ }^{1 a}$
Noelia Yesica Martínez Cántaro ${ }^{2 a b}$
\end{abstract}

iD https://orcid.org/0000-0001-5820-2228

iD https://orcid.org/0000-0003-0626-1773

Correspondencia: jennisse_16@hotmail.com

\section{Resumen}

La mordida cruzada posterior unilateral es un tipo de maloclusión que se caracteriza por una deficiencia de arco, en la cual pueden presentarse asimetrías en el arco superior o inferior. Uno de los abordajes es la técnica de arco segmentado, la cual nos permite predecir los resultados minimizando el tiempo de tratamiento y mejorando la comodidad del paciente. Este reporte de caso tiene como objetivo describir el manejo clínico de un retratamiento ortodóntico de mordida cruzada posterior unilateral utilizando la técnica de arco segmentado, mediante el uso de dispositivos como ATP, arco lingual, bite plane, finalizando con arcos MEAW. Se concluye con la importancia del correcto diagnóstico y la elección de una adecuada técnica para el tratamiento de una mordida cruzada posterior unilateral. Esta técnica es una herramienta importante que todo ortodoncista debe considerar para definir su plan de tratamiento y así evitar efectos secundarios y un tratamiento prolongado.

Palabras clave: mecánica, arco segmentado, mordida cruzada posterior

\begin{abstract}
The unilateral posterior crossbite is a type of malocclusion that is characterized by an arch deficiency, in which asymmetries in the upper or lower arch may occur. One of the approaches is the segmented arch technique, which allows us to predict the results, minimizing treatment time and improving patient comfort. This case report aims to describe the clinical management of a unilateral posterior crossbite orthodontic retreatment using the segmented arch technique, through the use of devices such as ATP, lingual arch, bite plane, ending with MEAW archwires. It concludes with the importance of a correct diagnosis and the choice of an adequate technique for the treatment of a unilateral posterior crossbite. This technique is an important tool that every orthodontist should consider to define their treatment plan and thus avoid side effects and prolonged treatment.
\end{abstract}

Keywords: mechanics, segmented arch, posterior crossbite

\footnotetext{
${ }^{1}$ Investigador independiente. Tacna, Perú.

${ }^{2}$ Universidad Nacional Jorge Basadre Grohmann. Escuela Profesional de Odontología. Tacna, Perú

${ }^{a}$ Especialista en Ortodoncia y Ortopedia maxilar

${ }^{\mathrm{b}}$ Maestro en Estomatología
} 


\section{Introducción}

La mordida cruzada posterior corresponde a una maloclusión en el plano transversal del maxilar; definiéndose como la alteración en la correcta articulación de las cúspides palatinas de molares y premolares superiores con las fosas de molares y premolares inferiores. ${ }^{1-3}$

La prevalencia de la mordida cruzada posterior es de $15.8 \%$, siendo mayor en la dentición permanente en un $35.7 \%$, la mordida cruzada posterior unilateral en $9.5 \%$ y bilateral en un $6.2 \%$.

Dada la frecuencia de alteraciones transversales que se presenta en la práctica diaria, vemos la necesidad de realizar un buen diagnóstico diferencial de las mismas para poder adecuar nuestros tratamientos de la forma más eficaz y con los resultados más estables posibles.

La mordida cruzada unilateral se caracteriza por una deficiencia de arco y algunas veces por asimetrías en el arco superior o inferior. ${ }^{4}$

Existen diferentes técnicas de tratamiento para resolver este tipo de maloclusión. En este reporte se describirá la técnica de arco segmentado como una de las opciones para tratar una mordida cruzada posterior unilateral.

La técnica de arco segmentado fue desarrollada por el Dr. Charles Burstone en 1962. Esta técnica actúa de forma segmentada permitiendo solucionar los problemas por grupos de dientes; las bases de la técnica de arco segmentado se rigen por la mecánica para predecir los movimientos dentarios. ${ }^{5-7}$ Esta técnica es particularmente adecuada para los casos complejos asimétricos transversales. ${ }^{8-10}$

El fundamento de la técnica es la segmentación; que significa la consolidación de los dientes en unidades, lo que permite utilizar cada bloque como una raíz de diente, como inserción. A partir de esta segmentación se divide los bloques en una unidad activa (la que se mueva) y una unidad reactiva o anclaje. ${ }^{11}$

Es así que no emplea arcos continuos de níquel titanio o acero inoxidable como se suele utilizar en la técnica de arco continuo; sino que, en lugar de ello, emplea arcos de beta titanio, que permiten realizar dobleces sin que el alambre pierda sus propiedades mecánicas. ${ }^{12}$

Esta técnica permite conseguir los objetivos de tratamiento de casos complejos en el menor tiempo posible, empleando cualquier tipo de prescripción y mediante el uso de diferentes dispositivos específicos para resolver problemas biomecánicamente complejos a través del uso de arco segmentado; es por ello que en el presente trabajo se utilizó dos dispositivos, que son el arco lingual y el arco transpalatino, para resolver el caso clínico de mordida cruzada posterior unilateral evitando efectos secundarios que dilaten el tratamiento y que ocasionen un daño biológico.

\section{Caso clínico}

Paciente de sexo femenino, de 15 años y 11 meses de edad y raza mestiza acude a consulta con el motivo de "Quiero continuar mi tratamiento".

Al examen clínico extraoral se determinó un biotipo mesofacial, simétrico, competencia labial, labios gruesos y normotónicos, de perfil total convexo, plano mandibular normodivergente, ángulo nasolabial y mentolabial disminuido, perfil del tercio inferior convexo. En el análisis de la sonrisa se observó asimetría de tipo baja, arco de sonrisa consonante, línea del labio superior media; presenta canteo oclusal hacia la derecha, línea media dentaria no coincidente, presenta diastemas (Figura 1). 


\section{Figura 1}

Fotografías extraoales iniciales

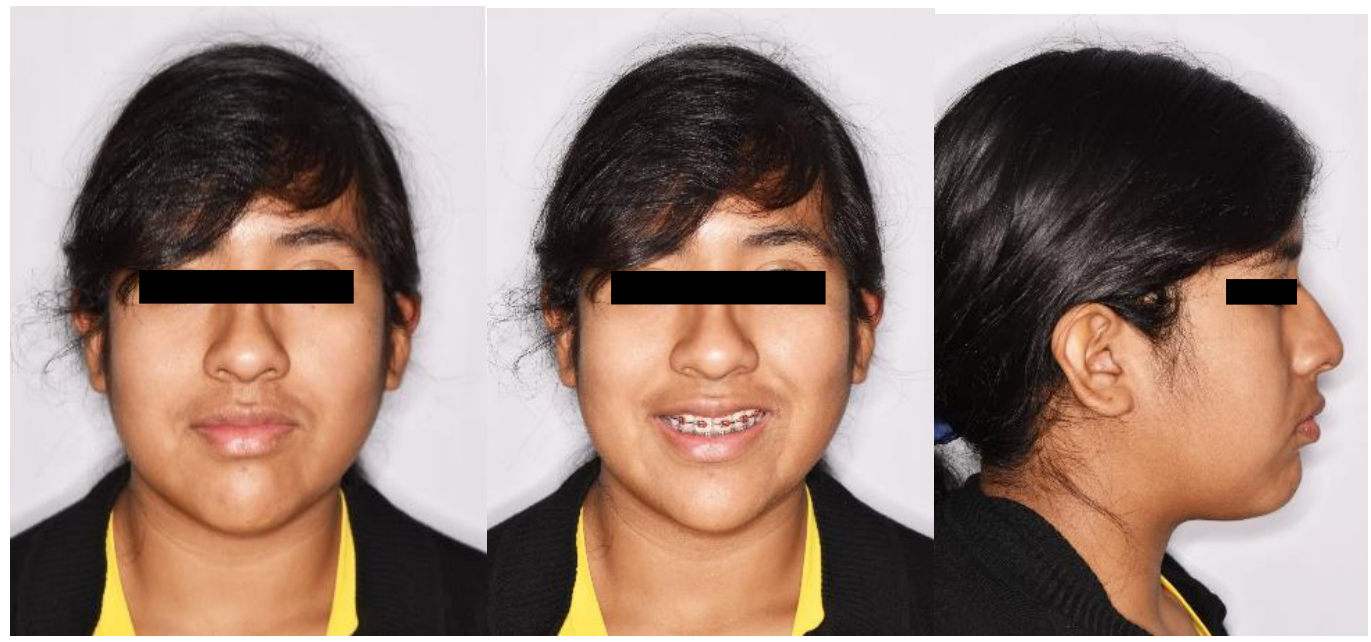

Al examen clínico intraoral se observó un bajo riesgo de caries, encías saludables, maloclusión según Angle de Clase I con relaciones molares y caninas de Clase I; a su vez presentaba mordida cruzada posterior unilateral localizada en las piezas 47/17-16, 46/16, curva de Spee de $2 \mathrm{~mm}$, forma de arco superior e inferior parabólico, ausencia de las piezas 14, 24, 34, 44, OJ de $4 \mathrm{~mm}$, OB de $4 \mathrm{~mm}$, línea media dentaria no coincidente, línea media superior alineada a la línea media facial e inferior $0.5 \mathrm{~mm}$ a la izquierda de la misma (Figura 2 ).

\section{Figura 2}

Fotografías intraorales iniciales

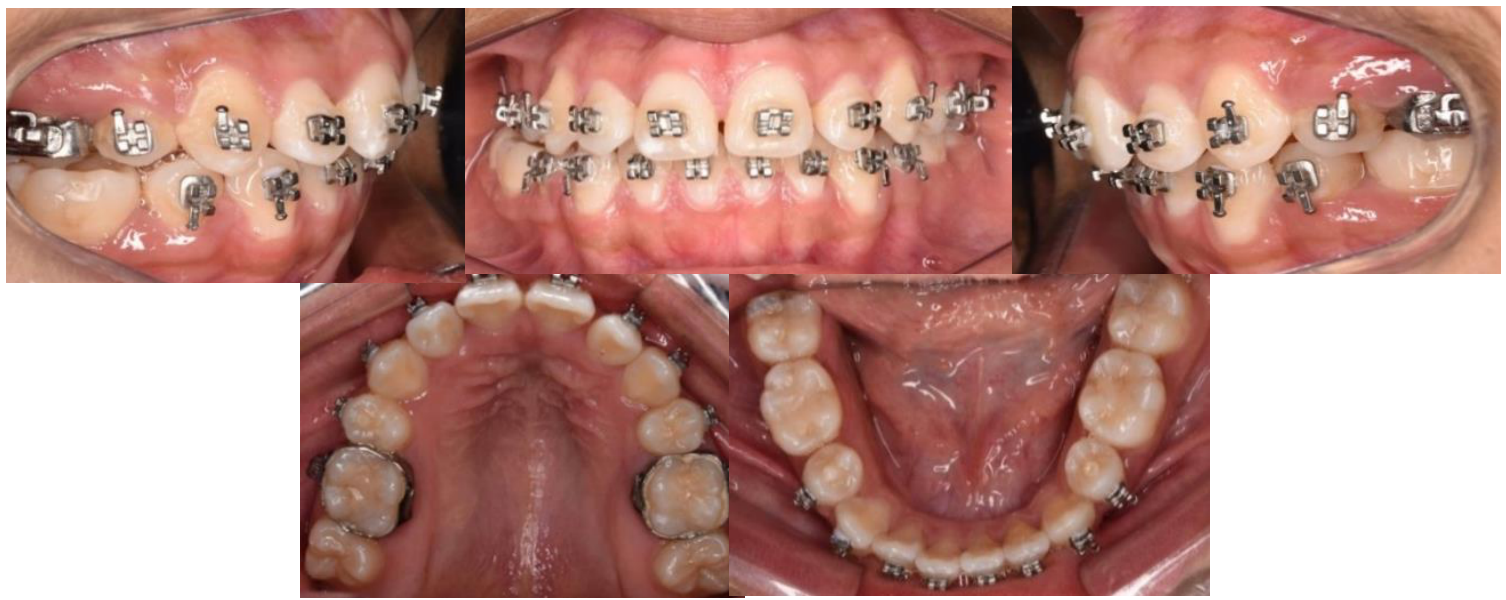

El análisis radiográfico presentó, en la radiografía panorámica, cóndilo derecho engrosado, estructuras óseas de características normales, raíces no paralelas en piezas 22, 25, 35, cornete derecho reducido, ausencia de las piezas 14, 24, 34, 44. Piezas 18, 28, 38, 48 en estadio de Nolla 6.

El trazado cefalométrico de la radiografía lateral de cráneo nos indica una relación esquelética de Clase II $\left(\right.$ ANB $\left.=6^{\circ}\right)$ con normoposición maxilar $\left(\right.$ SNB $\left.=83^{\circ}\right)$, crecimiento neutral-normodivergente $(62 \%)$, maloclusión Clase I de Angle con asimetría transversal unilateral posterior derecha (esquelética), 
incisivos superiores palatinizados y retruidos, incisivos inferiores retruidos. El marco dental nos indica discrepancia superior de $3.5 \mathrm{~mm}$, discrepancia inferior de $0.5 \mathrm{~mm}$ (Figura 3).

\section{Figura 3}

\section{Radiografías y cefalometría}

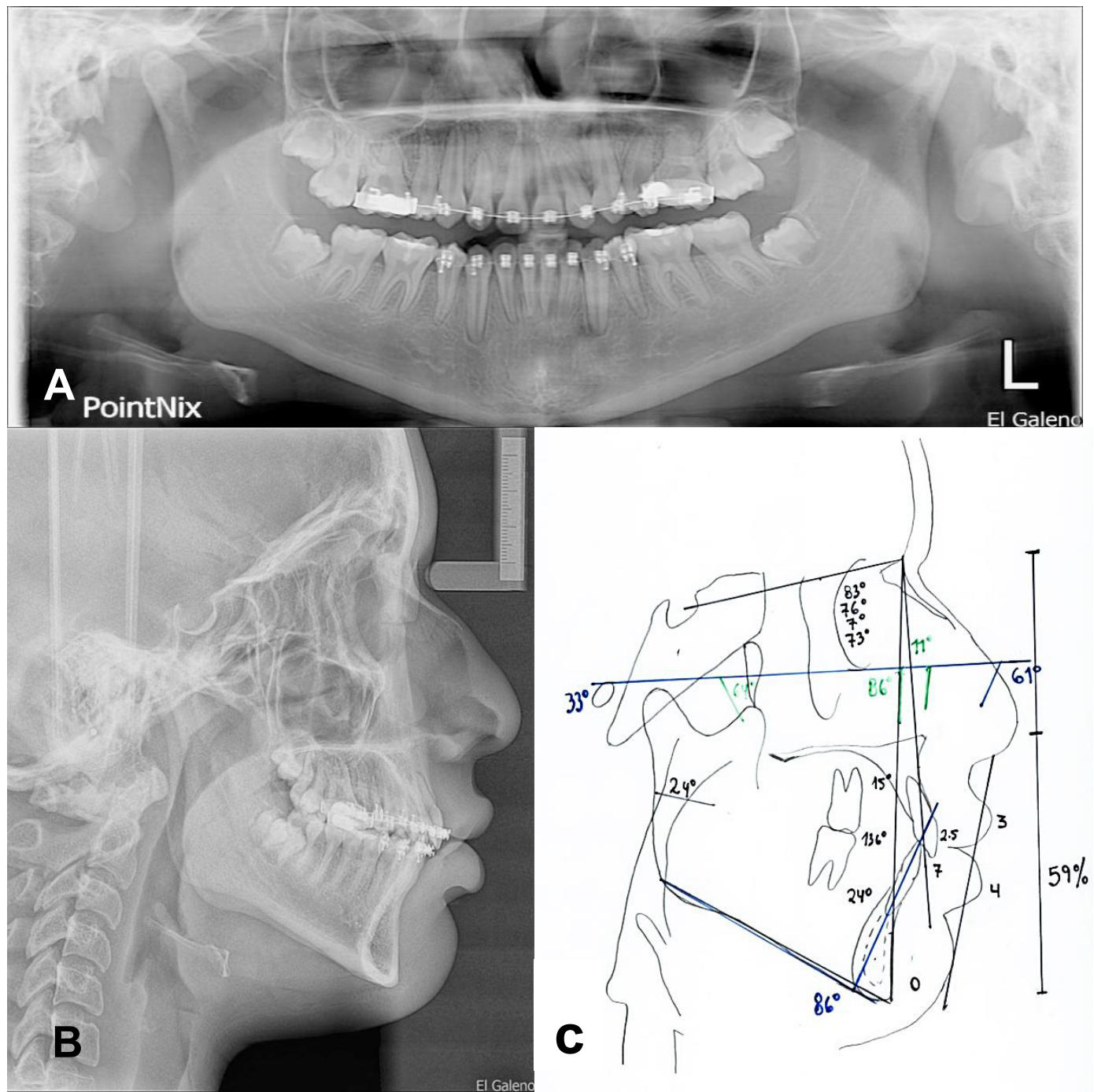

A. Radiografía panorámica inicial. Downs.
B. Radiografía lateral inicial.

C. Cefalometría de Steiner, Tweed y

\section{Evolución del caso}

Siendo un retratamiento ortodóntico, las piezas dentarias se encontraban alineadas por lo que se procedió a corregir la mordida cruzada posterior unilateral derecha.

El tratamiento inició con la confección de bandas en los primeros molares superiores e inferiores con tubos triples y dobles, respectivamente; también se soldaron cajas linguales. Se procedió a confeccionar ATP y arco lingual removible con alambre $0.08 \mathrm{Ss}$. Como arco base se utilizó alambre $0.016 \mathrm{NiTi}$ superior y $0.014 \mathrm{NiTi}$ inferior, a la vez se confeccionó un bite plane en el cuadrante 2 con acrílico de curado rápido, registrando las caras oclusales antagonistas; el uso del bite plane se 
prioriza para levantar la mordida y descruzar el lado opuesto. EI ATP fue activado para expandir el arco superior y el arco lingual fue activado para comprimir el arco inferior. Se pegaron tubos adhesivos en las piezas 17, 47 y un botón adhesivo a nivel de la cara palatina de la pieza 17. Se utilizó elásticos cruzados 3/16 de 6 onzas en piezas 16/46, 17/47 para descruzar la mordida cruzada posterior unilateral (Figura 4).

\section{Figura 4}

Procedimiento para corregir la mordida cruzada posterior unilateral

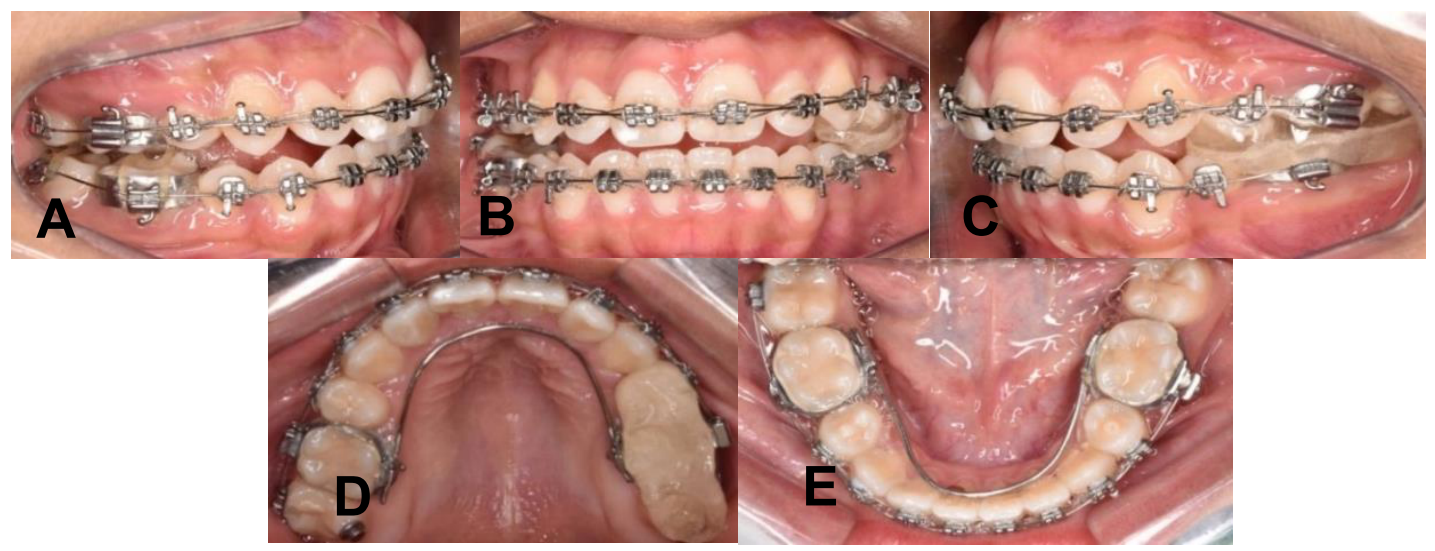

A. Elásticos cruzados $3 / 16$ de 6 onzas en piezas 16/46. B. Vista frontal del levante de mordida. C. Instalación del bite plane. D. Activación de ATP. E. Activación del arco lingual.

Después de 2 meses obtuvimos la corrección de mordida cruzada posterior unilateral, por lo que procedimos al retiro del bite plane y a esperar el asentamiento de mordida, arco base superior e inferior 0.016 NiTi (Figura 5).

\section{Figura 5}

\section{Corrección de la mordida cruzada}

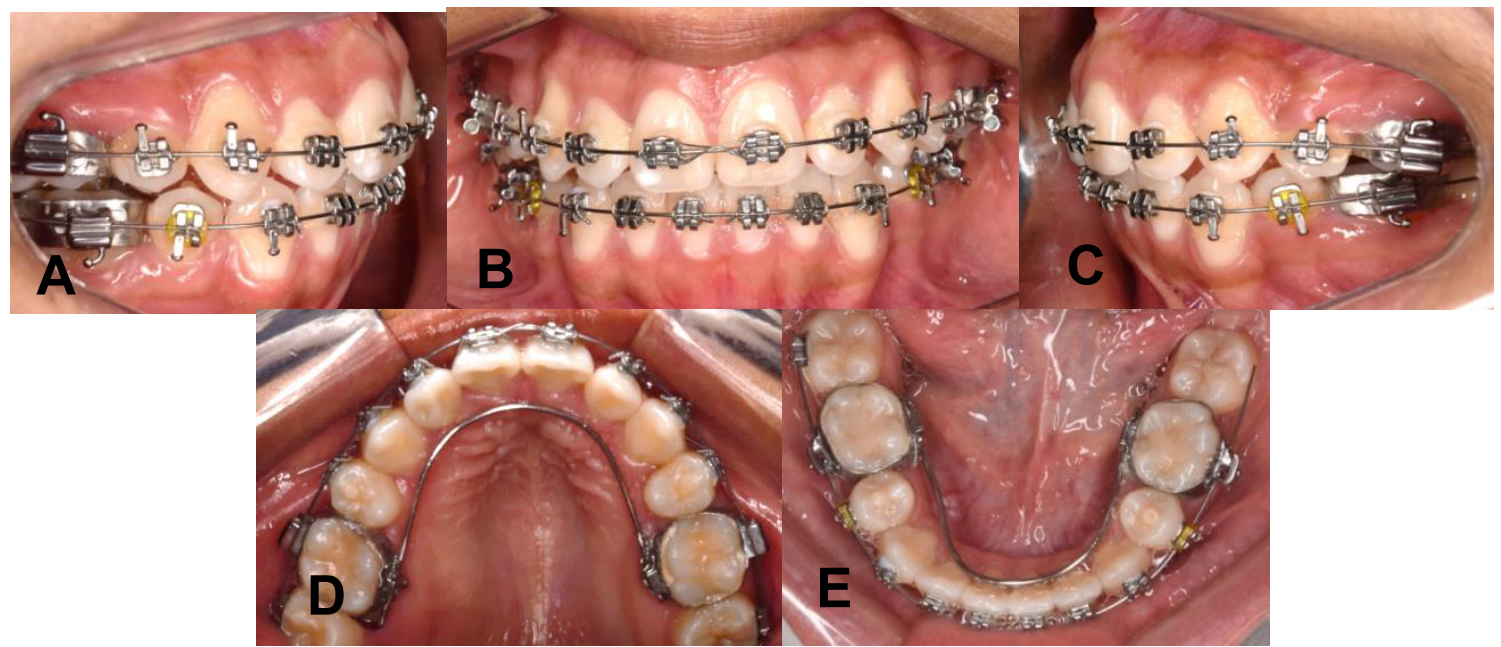

A. Vista lateral derecha.

B. Vista frontal.

C. Vista lateral izquierda.

D. Vista oclusal superior. E. Vista oclusal inferior. 
Se continuó con la confección de arcos MEAW con alambre elgiloy azul 0.016 " x 0.022" con elásticos de Clase I. Activación de arco MEAW con torque compensatorio positivo a nivel de las piezas 17, 16, 15 y torque compensatorio negativo a nivel de las piezas 46, 47.

Se recurrió al uso de elásticos intermaxilares 3/16 de 6 onzas de Clase I (1,2 con 2) para llave canina, luego siguió la eliminación de interferencias, activación step down en pieza 46, eliminación de contacto, continuando con elásticos intermaxilares de Clase I y lateral para corrección de línea media.

Se procedió al seccionamiento de arcos MEAW a nivel distal de las piezas 13 y 43 para mejorar el engranaje posterior con elásticos intermaxilares de Clase I, y la obtención de una oclusión fisiológica (Figura 6).

\section{Figura 6}

Evolución del caso

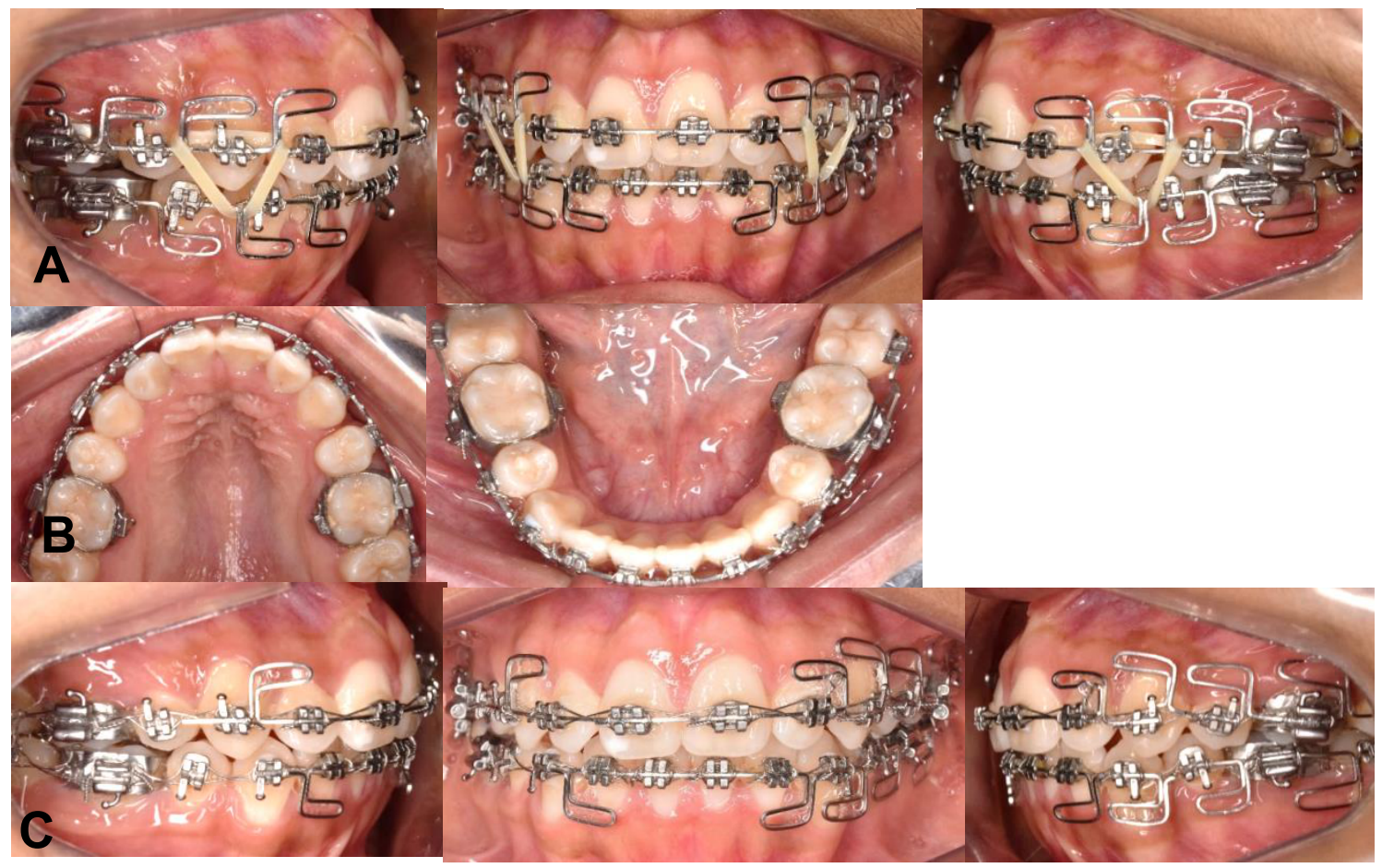

A. Confección de arcos MEAW con alambre elgiloy azul 0.016" x 0.022", con elásticos de Clase I. B. Retiro de ATP y arco lingual. C. Seccionamiento de arcos MEAW a nivel distal de las piezas 13 y 43.

También se evaluaron los aspectos funcionales de la paciente, la misma presentaba guías caninas en lateralidad en ambos lados y desoclusión posterior en protrusiva. Los resultados obtenidos fueron la corrección de la mordida cruzada posterior unilateral, se cerraron los espacios, el OB disminuyó a $3 \mathrm{~mm}$ y se alineó la línea media. Se mejoró la relación molar, el OJ disminuyó a $2 \mathrm{~mm}$ (Figura 7). Finalmente, luego de 15 meses, fueron retirados los brackets e instaladas las contenciones, siendo removible en la arcada superior; y fija, en la inferior (Figura 8). 


\section{Figura 7}

Término del tratamiento
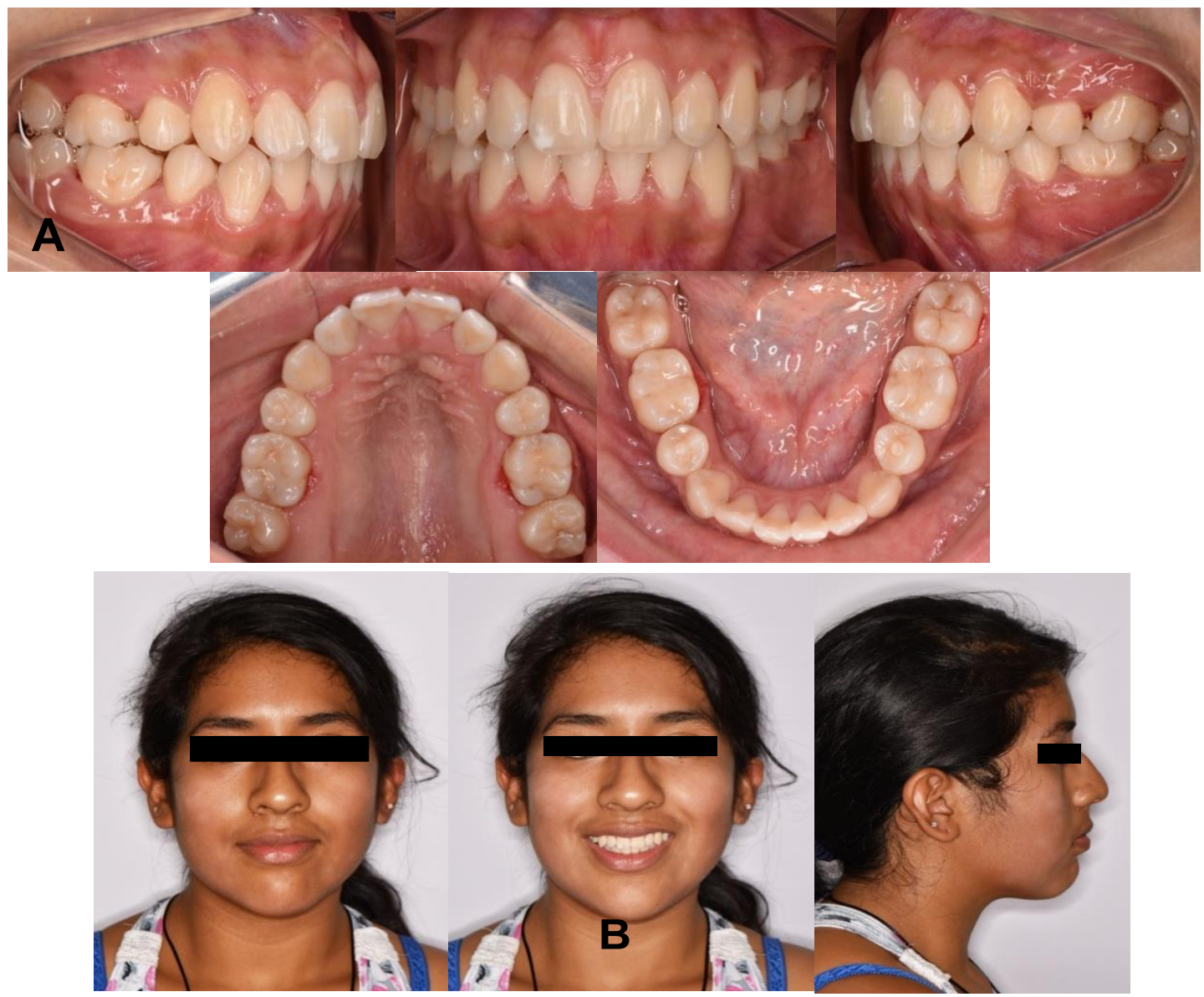

A. Fotografías intraorales finales.

B. Fotografías extraorales finales.

\section{Figura 8}

Instalación del aparato de contención Wrap around removible superior (alambre 0.09 Ss) y contención fija inferior de 35 a 45 con alambre 0.07 Ss.

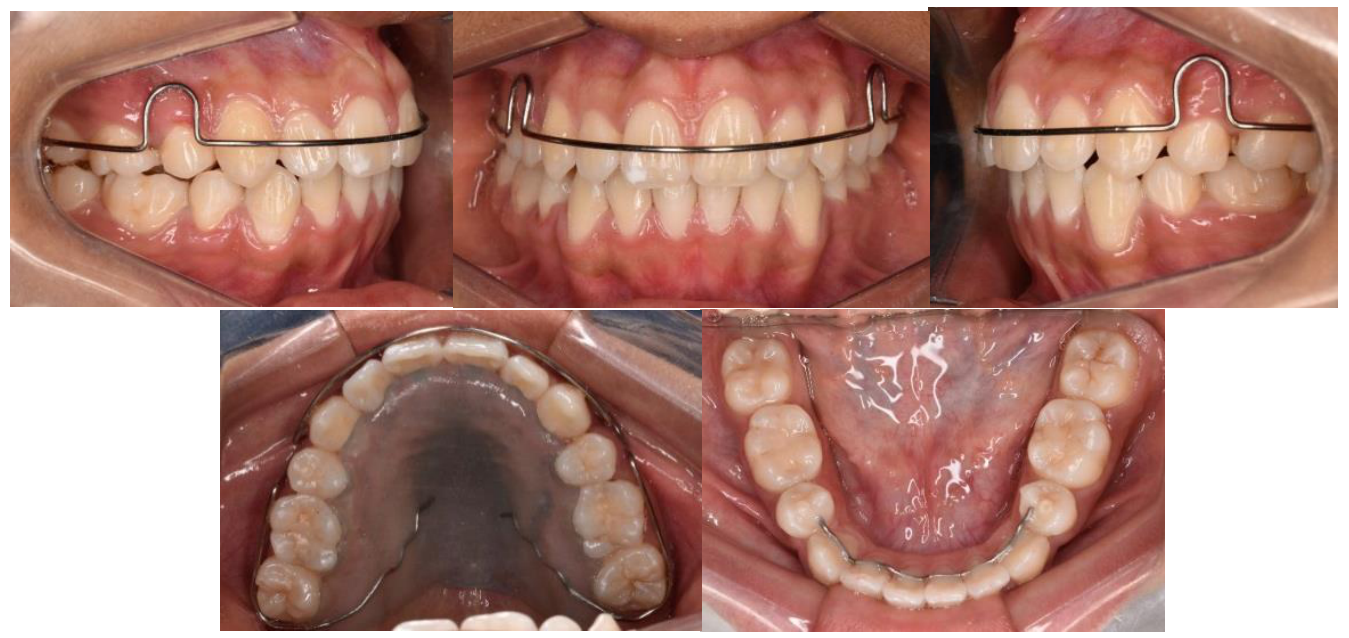


En el análisis del modelo superior final hubo un aumento en la distancia intermolar de $53 \mathrm{~mm}$ a 54 $\mathrm{mm}$ y de la correspondencia molar de 36 a $37 \mathrm{~mm}$. En el caso del análisis del modelo inferior final se redujo la distancia intermolar de $51 \mathrm{~mm}$ a $47 \mathrm{~mm}$ y de la correspondencia molar de $42 \mathrm{~mm}$ a $39 \mathrm{~mm}$.

En el trazado cefalométrico final se mantuvo la Clase II esquelética: las medidas esqueléticas presentaron ligeros cambios, ya que el tratamiento fue ortodóntico.

\section{Figura 9}

Estudios al término del caso

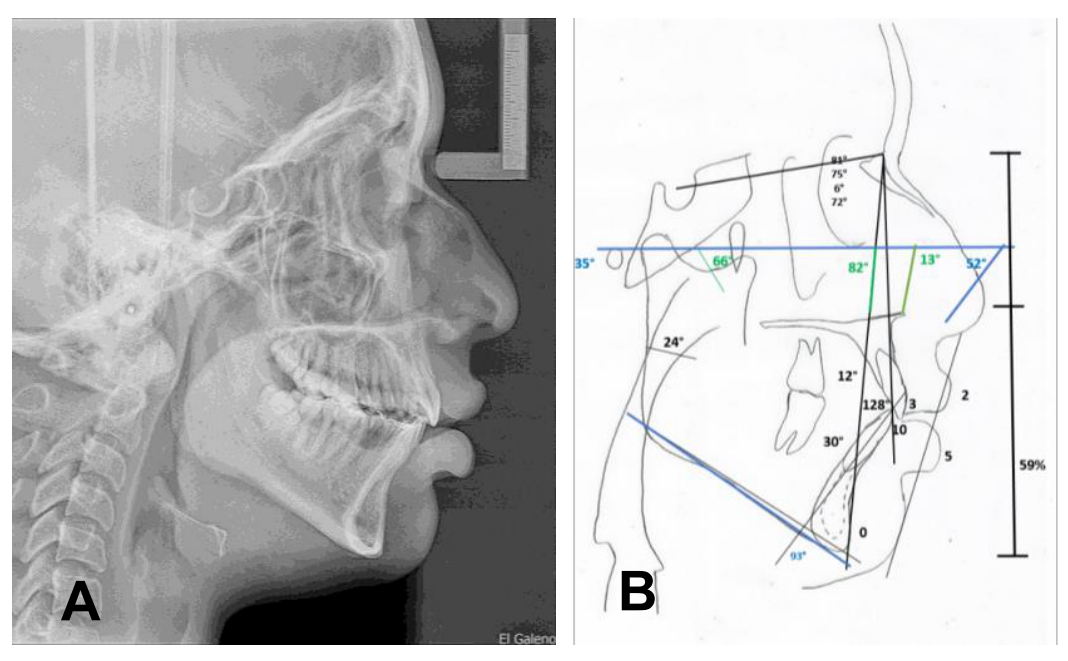

A. Radiografía lateral final. B. Cefalometría final.

\section{Discusión}

La utilización de la técnica de arco segmentado es una opción en ortodoncia que nos permite resolver este tipo de maloclusión, evitando efectos secundarios y minimizando el tiempo de tratamiento. Ya que el caso clínico es un retratamiento ortodóntico, esta técnica utiliza diferentes aditamentos como ATP y arco lingual, anexando ligas (elásticos) intermaxilares cruzados.

En el presente reporte de caso el uso de la técnica de arco segmentado mostró una expansión e inclinación dentoalveolar, un aumento de distancia intermolar de $1 \mathrm{~mm}$ y correspondencia molar de $1 \mathrm{~mm}$. Este resultado está de acuerdo con otros estudios que muestran cómo el efecto más importante de la NPE fue una expansión dentoalveolar controlada y una leve remodelación ósea. ${ }^{13}$ Como se sugiere en muchos artículos, el efecto más importante de la NPE fue sobre posición transversal molar. Clambotti et al. mostraron un aumento en el ancho intermolar de $6.26 \mathrm{~mm}$ en paciente con NPE. Donohue et al. evaluaron un aumento de la distancia de los molares superiores de $7.8 \mathrm{~mm}$ y $5.9 \mathrm{~mm}$ en los primeros molares. ${ }^{14}$

En un estudio realizado por Graziano M. la NPE-2 generó una posición vestibular efectiva de los molares superiores de $2.3 \mathrm{~mm}$ en los molares y $2.9 \mathrm{~mm}$ en la cabeza. Los autores mostraron que no se observó una modificación importante en los molares inferiores, tanto en la evaluación cefalométrica ( $0.8 \mathrm{~mm}$; NS) como en los moldes $(1.4 \mathrm{~mm}$; NS). Se observó una expansión de los dientes en los primeros premolares $(1.6 \mathrm{~mm})$ y en los caninos $(1.4 \mathrm{~mm}) .^{13}$

En nuestra paciente se resolvió la mordida cruzada posterior unilateral en dos meses en una dentición permanente; otros estudios, como la de Lars Granath y Sven-Olof Peterson, mencionan que las mordidas cruzadas unilateral funcional se resolvieron en 15 días en niños de dentición primaria y la distancia de los segundos molares aumentó la anchura transversal de 1.9 a $3.8 \mathrm{~mm}$ y 
entre los caninos por 1.9 a $3.3 \mathrm{~mm} .{ }^{15}$ Por todo esto es importante el tratamiento precoz de la mordida cruzada posterior para minimizar asimetrías esqueléticas mayores, así como para evitar alteraciones estéticas y funcionales. El tratamiento precoz evitará la aparición de patología en la ATM, alteraciones en la musculatura, aparición de hábitos deletéreos como el bruxismo y problemas estéticos. ${ }^{9}$

\section{Conclusiones}

El diagnóstico correcto de las mordidas cruzadas es relevante, porque si estas tienen compensaciones dentoalveolares al realizar una expansión, tendremos una mordida en tijera; además, detectada la misma puede ser tratada en una fase precoz.

Los cambios dentoalveolares inducidos por la aplicación de la técnica segmentada muestran una efectividad en la corrección de una mordida cruzada posterior unilateral, siendo el manejo muy sencillo. La ventaja de la técnica segmentada reside en la precisión y rápido desplazamiento en los tres planos del espacio. Esta técnica está diseñada para ofrecer fuerzas relativamente constantes y óptimas con el objetivo de un control total sobre los dientes que van a ser movidos.

\section{Referencias}

1. Lorente P. Clasificación y tratamiento de las maloclusiones transversales. Rev Esp Ortod 2002; 42: 179-81.

2. Theodosia Bartzela, Irmtrud J. Long-term Stability of Unilateral Posterior Crossbite Correction. Angle Orthod.2007; 77(2): 237- 243.

3. Arno L, André W, Daltro E, Gerson Luis U, Luciane M, Carla D. et al. Mordida cruzada posterior: uma classificacao mais didática. Dental Press Ortodon Ortop Facial. 2008; 13(2): 146-158.

4. Kahraman Gungor, Lale T, Emine K. Prevalence of Posterior Crossbite for Orthodontic Treatment Timing. The Journal of Clinical Pediatric Dentistry. 2016; 40(5): 422-424.

5. Sakima M. Técnica do Arco Segmentado de Burstone. Dental Press Ortodon Facial. 2000;(2).

6. Charles J. Burstone C. The mechanics of the segmented arch tecniques. 1966;36(2):99-120.

7. Evelyn Roxana P M. Técnica de arco segmentado. Universidad Inca Garcilazo de la Vega:2018.

8. Philippe Tuil, Guilles B. La segmentation de l'arc, une réponse aux difficultés de traitment de cas atypiques. Orthod Fr. 2019; 90:65-74.

9. Bartzela T, Jonas I. Long-term stability of unilateral posterior crossbite correction. Angle orthod. 2007; 77(2):237-43.

10. María Concepción Martín Alvaro. Características neuromusculares de la mordida cruzada posterior unilateral: Estudio electromiográfico, kinesiográfico y tomográfico. Tesis doctoral Universidad Complutense de Madrid. 1999.

11. Stanley Braun, Michael RM. Rationale of the segmented approach to orthodontic treatment. AM J Orthod Dentofac Orthop. 1995; 108: 18.

12. Sergei Godeiro Fernandes RC, Alexandre R, Hallissa S, André Wilson M. Segmented arch or continuous arch technique? A rational approach. Dental Press J Orthod. 2014; 19(2): 126 141.

13. Graziano Montaruli, ML, LLR, CC, LLM, DC, et al. Titanium Palatal Expander (NPE-2) in Young Adults. 2016; Dent Oral Disord Ther 4(2): 1-6. 
14. Donohue VE, Manshman LA. WINchester L.J. Una comparación clínica del dispositivo quadhelix y el expansor palatino de niquel-titanio (bucle tándem): una investigación preliminar prospectiva. Eur J orthod. 2004; 26(4): 411-420.

15. Lars Granath, Olof P. A modified palatal arch for treatment of unilateral functional cross-bite in the primary dentition. Europan Journal of Orthodontics.1994; 16. 35-40. 